\title{
Can the Network be the Al Accelerator?
}

\author{
Davide Sanvito \\ Politecnico di Milano, \\ NEC Laboratories Europe \\ davide.sanvito@polimi.it
}

\author{
Giuseppe Siracusano \\ NEC Laboratories Europe \\ giuseppe.siracusano@neclab.eu
}

\author{
Roberto Bifulco \\ NEC Laboratories Europe \\ roberto.bifulco@neclab.eu
}

\begin{abstract}
Artificial Neural Networks (NNs) play an increasingly important role in many services and applications, contributing significantly to compute infrastructures' workloads. When used in latency sensitive services, NNs are usually processed by CPUs since using an external dedicated hardware accelerator would be inefficient. However, with growing workloads size and complexity, CPUs are hitting their computation limits, requiring the introduction of new specialized hardware accelerators tailored to the task. In this paper we analyze the option to use programmable network devices, such as Network Cards and Switches, as NN accelerators in place of purpose built dedicated hardware. To this end, in this preliminary work we analyze in depth the properties of NN processing on CPUs, derive options to efficiently split such processing, and show that programmable network devices may be a suitable engine for implementing a CPU's NN co-processor.
\end{abstract}

\section{CCS CONCEPTS}

- Networks $\rightarrow$ Programmable networks; In-network processing; • Computing methodologies $\rightarrow$ Machine learning; $\bullet$ Computer systems organization $\rightarrow$ Neural networks;

\section{KEYWORDS}

Computation offloading; Programmable switches; SmartNIC

\section{ACM Reference Format:}

Davide Sanvito, Giuseppe Siracusano, and Roberto Bifulco. 2018. Can the Network be the AI Accelerator?. In NetCompute'18: Morning Workshop on In-Network Computing, August 20, 2018, Budapest, Hungary. ACM, New York, NY, USA, 6 pages. https://doi.org/10.1145/3229591.3229594

\section{INTRODUCTION}

NNs are a tool for machine learning that has recently outperformed other approaches in classification tasks, for instance in computer vision. The widespread application of the technique in different fields is significantly impacting datacenters' workloads, which now dedicate a large amount of computing resources to the task of executing NNs [5].

A NN workload has two phases. The training phase is used to learn the NN's weights, fitting the NN to the specific task at hand. Once trained, the NN is used for the inference phase. Usually the

Permission to make digital or hard copies of all or part of this work for personal or classroom use is granted without fee provided that copies are not made or distributed for profit or commercial advantage and that copies bear this notice and the full citation on the first page. Copyrights for components of this work owned by others than ACM must be honored. Abstracting with credit is permitted. To copy otherwise, or republish, to post on servers or to redistribute to lists, requires prior specific permission and/or a fee. Request permissions from permissions@acm.org.

NetCompute'18, August 20, 2018, Budapest, Hungary

(c) 2018 Association for Computing Machinery.

ACM ISBN 978-1-4503-5908-5/18/08 . \$ \$15.00

https://doi.org/10.1145/3229591.3229594 training is a purely offline task, computationally expensive but performed with relatively low frequency. The inference phase is much lighter, but performed many times with potentially very strict latency constraints, since the NN's prediction may be used as part of an online service.

Being computationally expensive, the training phase is generally performed by specialized hardware accelerators, for instance general purpose GPUs. These devices can perform parallel computation on large amounts of data, making computation quicker and cheaper, therefore economically viable. Nonetheless, the use of an accelerator incurs some overheads. First, data needs to move within a compute node, i.e., from the general purpose CPU, where it is preprocessed, to the accelerator that is attached to, e.g., the machine's PCIe bus. Second, current accelerators provide higher efficiency when performing parallel computation on larger batches. The additional data movements and the need for batching finally affect the overall end-to-end latency. As a result, it is usually more efficient to use of regular CPUs for the NN inference phase, especially when handling latency sensitive workloads [6].

Unfortunately, the end of Dennard's scaling is challenging the ability to further scale CPUs' computing power [4]. As a matter of fact, Facebook reports that more efficient single CPU machines are replaced with less efficient but more powerful multi-CPUs machines to match required performance levels, for some current machine learning workloads [6]. Likewise, Google already designed and deployed the TPU, a custom ASIC dedicated to NN inference workloads [9].

Given this premise, as online and time sensitive inference workloads grow, it seems we are left with only two options to handle them: i) using today's accelerators for online NNs processing, accepting the associated inefficiencies; ii) or going through the effort and complexities of designing and deploying new, special-purpose accelerators dedicated to $\mathrm{NN}$ inference.

We instead look to a third alternative leveraging network devices to assist the computation performed on a machine's CPUs. In other words: Can the network be the accelerator?

Our work yields from two observations. First, Network Interface Cards (NICs) and switches are increasingly incorporating programmable features. Such programmability is surely helpful to address the implementation of typical network packet processing functions, but it also gives a great chance to perform packet processing in a way that benefits end-hosts applications $[3,8,13]$. That is, the ability to program network devices operations suddenly changes a dumb pipe, which only moves data, into a processing pipeline that transforms data as it flows. Second, these devices sit already on the data path, which minimizes the need to perform additional expensive data movements and reduces the end-to-end processing latency.

Towards this vision, we provide three contributions: 


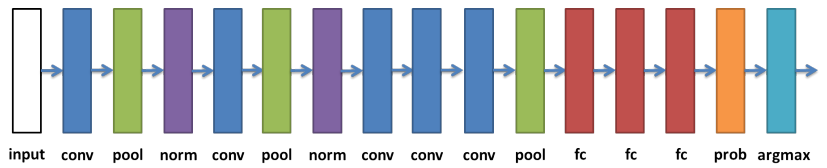

Figure 1: The sequence of layers of AlexNet [12]

i) we analyze in details the computation required to perform inference with a deep convolutional $\mathrm{NN}$, and profile its execution on general purpose CPUs for different machine configurations. Our analysis suggests that CPUs cannot be leveraged at full speed when processing fully connected layers, mainly for the need to stall the CPU's pipeline due to memory cache misses.

ii) Building on this observation and leveraging the NN's layeredstructure, we analyze options for partitioning a NN processing in order to offload (a subset of) NN's layers from the CPU to a different processor. We find that it is possible to define a NN execution split that minimizes the overhead of moving computation from a processor to a different one.

iii) Our last contribution is to evaluate under which conditions NICs and switches are suitable to work as CPUs co-processors for the processing of NNs. We do so designing BaNaNa Split, a solution that leverages recent techniques to perform NN models quantization and execute such quantized models on both network processor-based SmartNICs and programmable switching chips.

While still at an early stage, BaNaNa Split shows that already available network hardware may help handling a large fraction of currently reported datacenter NN inference workloads.

We believe our findings may fuel the discussion on both NN processing infrastructures and network devices research.

\section{BACKGROUND}

\subsection{Artificial Neural Networks}

A NN is a collection of interconnected neurons organized in layers. A neuron takes inputs (called activations) from neurons of previous layer and sends its output to neurons in the next layer. In general, one can think of a layer as a blackbox that takes an activations vector and transforms it in an output vector. The number of layers, their types and the number of neurons per layer are the hyperparameters of a NN that finally impact the ability of the network to perform the task at hand. For example, a fully connected layer ( $\mathrm{fc}$ ) has neurons that take as activations all the outputs of a previous layer's neurons, and that provide their outputs to all the next layer's neurons. A network with only fc layers is called a multi-layer perceptron (MLP). Deep NNs have more than three layers and among those, convolutional $\mathrm{NN}(\mathrm{CNN})$ have recently achieved outstanding performance in some classification tasks, in particular for computer vision. Fig. 1 shows the sequence of layers of the AlexNet [12] CNN. The convolutional (conv) and fc layers have weights, called the parameters of the NN, associated with each input of a layer's neuron. The remaining layers only perform some fixed activations transformation and therefore do not have weights.

It's interesting to notice the three fcs towards the end of AlexNet model, since when taken alone they are in all similar to a MLP model. This is a common feature of all the CNNs. Other types of NNs, such as recurrent $\mathrm{NNs}$, have a different structure and will not be covered in this paper.

In terms of inference workload composition, MLPs are by far the most common NNs, followed by RNN and CNN, constituting $61 \%, 29 \%$ and $5 \%$ of the workload, respectively, according to [9]. Since MLPs can be thought as a special case of CNNs, in the rest of the paper we will focus on CNNs, and in particular on AlexNet. In effect, recent NNs may have a bigger number of layers, however, since additional layers are just repetitions of those presented in Fig. 1, the AlexNet model remains a valid example while simplifying exposition given its relative simplicity.

\subsection{Neural Network Accelerators}

NN accelerators, such as general purpose GPUs (GPGPUs), are commonly deployed as an additional hardware board attached to a machine's PCIe bus. These devices generally use a Single Instruction Multiple Data (SIMD) paradigm to perform large parallel computations. Thus, they are efficiently used when a given task is executed on a large amount of data, for which batching is typically employed.

Therefore, from a system perspective, the use of an accelerator to perform NN inference unfolds as follows. First, a request containing the inference task is received through the machine's NIC and sent over the PCIe bus to the RAM. Second, the CPU performs preprocessing of the request to extract the NN input, and adds it to the current batch. Third, once the batch is composed, it is transferred to the accelerator where the inference is performed. Fourth, the result is copied back to main memory, over the PCIe bus. Finally, the CPU prepares the result for transmission to the network, i.e., placing it in network packets, and transfers it, once more over the PCIe bus, to the NIC. We expect a front-end server will receive such result and include it in a user-facing service.

The need for batching and the data movements over the PCIe bus add significant latency to the end-to-end processing of the $\mathrm{NN}$ inference. For instance, [9] reports that, in their specific settings, the batch size for a GPGPU has to be reduced from 64 to 16 to meet processing latency constraints. This forces the GPGPU to work at $37 \%$ of its peak performance (achieved with a batch of 64 in such case). Furthermore, even if Google designed and deployed an accelerator, i.e., the TPU, explicitly targeting inference workloads, in some cases the time to transfer data to/from the TPU can be as much as $71 \%$ of the time spent during processing.

\section{WORKLOAD ANALYSIS}

To understand the issues with NN accelerators, in this section we analyze the processing required by AlexNet during inference. We perform both a static analysis derived from the model structure and a runtime analysis of the system's resources used during the processing on CPUs.

\subsection{Structural analysis}

A NN layers' number of parameters, and the size of the activations and output vectors, is fixed for a given network model. Fig. 2a shows the size of the output vector of each AlexNet's layer (notice that the initial input size is represented by the left-most bar of the histogram). It's interesting to notice that some layers have output vectors bigger than their activations vectors (e.g. conv2 has an 

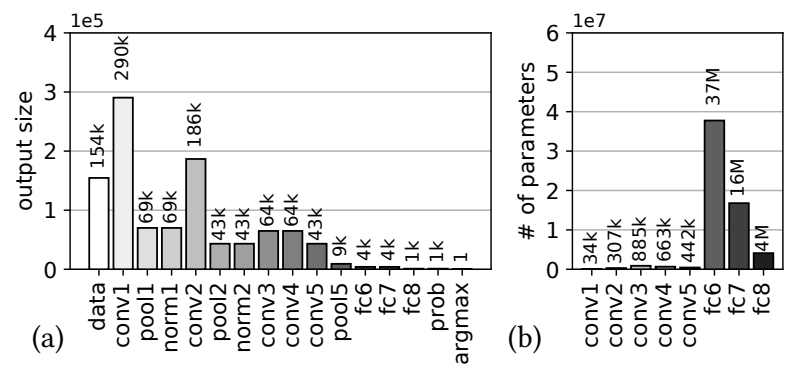

Figure 2: Per-layer output size and parameters

input with $69 \mathrm{~K}$ elements and produces $186 \mathrm{~K}$ elements), while for other layers this is reversed (e.g. pool1 layer sensibly reduces the number of output elements from $290 \mathrm{~K}$ to $69 \mathrm{~K})$. Also, it's worth noticing that $\mathrm{fc}$ layers have far smaller activations and outputs vectors than other layers, never bigger than $9 \mathrm{~K}$.

Interestingly, Fig. 2b shows that the number of parameters follows a different distribution: conv layers have at most $885 \mathrm{~K}$ parameters (conv3), while $\mathrm{fc}$ have far more parameters, topping to more than $37 \mathrm{M}(\mathrm{fc} 6)$.

\subsection{Runtime analysis}

We run AlexNet on a testbed composed of a dual-socket (NUMA) machine, running Linux kernel 4.10, with two CPUs Intel Xeon E5-2650 (8 cores@2.4GHz), hyperthreading disabled, and 16GB of RAM per socket. Each CPU has $32 \mathrm{~KB}$, for both L1 data and instruction caches, and $256 \mathrm{~KB}$ of L2 cache per core. $20 \mathrm{MB}$ of L3 cache are shared by all the CPU's cores. We use perf to collect performance counters, with a $10 \mathrm{~ms}$ polling interval and avoiding hardware counters multiplexing. AlexNet is implemented using the Caffe framework optimized for Intel processors [10], to take advantage e.g., of the CPU's vector processing features.

We instrument the system to collect both the total and per-layer inference latency. Furthermore, we collect hardware counters to measure the Instructions Per Cycle rate (IPC), the number of stalled cycles and the L1 (data), L2, L3 cache misses during execution. All the measurements are executed using a single isolated core on a dedicated CPU (i.e., all the other cores of the same CPU are idle and therefore the L3 cache is completely dedicated to the core running AlexNet).

Fig. 3 shows the time required to execute each layer of AlexNet when processing a single activation vector. Among the layers, conv2, conv 3 and conv 1 take the longer to execute, followed by fc6 and then the others.

Looking at the hardware counters, we derive the IPC achieved during the NN execution (solid line of Fig. 4). We discover the execution of the layers up to pool5 yields an IPC of 3.2, meaning that the CPU's pipeline is filled and that the processing is computationbound. Instead, the execution of the fc layers achieves an IPC below 1. This lower value is due to a high number of stalled cycles, caused by cache misses in all the cache levels. I.e., the system has to wait for data reads from the RAM. The dashed line of Fig. 4 shows the L3 cache misses increment for fc layers.

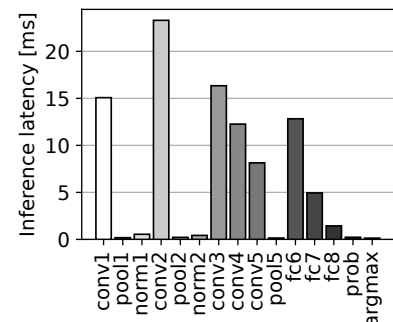

Figure 3: Per-layer inference latency

\begin{tabular}{|l|c|c|c|c|}
\hline Batch size & $\mathbf{1}$ & $\mathbf{1 6}$ & $\mathbf{3 2}$ & $\mathbf{6 4}$ \\
\hline $\begin{array}{l}\text { Batched proc. } \\
\text { latency [ms] }\end{array}$ & 96 & 1334 & 2724 & 5585 \\
\hline $\begin{array}{l}\text { Sequential proc. } \\
\text { latency [ms] }\end{array}$ & 96 & 1536 & 3071 & 6143 \\
\hline $\begin{array}{l}\text { Batched proc. } \\
\text { saving [\%] }\end{array}$ & - & 13.15 & 11.30 & 9.08 \\
\hline $\begin{array}{l}\text { Batched proc. } \\
\text { saving fc only [\%] }\end{array}$ & - & 69.74 & 73.75 & 80.32 \\
\hline
\end{tabular}

Table 1: Batched vs sequential inference processing time comparison
This performance is quickly explained by the type of computation performed by a fc layer, which does little reuse of the weights values. This, together with the large number of such layers' parameters, yields an inefficient use of the CPU's caches. For reference, just fc6's parameters need $151 \mathrm{MB}$ of memory, considering that each parameter is represented on $32 \mathrm{~b}$, i.e., 7 times the space available in the CPU's caches.

\subsection{Batching effects}

We re-run the previous test performing inference on a batch of 16 , 32,64 activations vectors. In Table 1 we compare the time taken to perform a batched execution with that required by a sequential execution of the activations contained in the batch. We can see that batching reduces the overall processing time, when compared to the sequential execution, with a reduction that ranges from $13 \%$, for a 16 vectors batch, to $9 \%$, for a 64 vectors batch. Indeed, this confirms that batching improves the processing efficiency.

To understand where the improvement lays, last row of Table 1 reports the effect of batching if we focus on the the processing time quota of $\mathrm{fc}$ layers. Moreover, in Fig. 5 we compare the relative contributions of the processing latency of each layer to the overall inference processing latency, when considering a non batched execution and one that uses a batch of 64 vectors. It's clear that $\mathrm{fc}$ layers get the most benefit from batching, moving from an overall contribution of $20 \%$ of the processing latency to just $4 \%$. In other words, most of the improvement on the processing efficiency due to batching is caused by an improvement in the efficiency of processing $\mathrm{fc}$ layers. This is confirmed by the analysis of the hardware counters, which show an increase of the IPC and a reduction of the cache misses. A behavior easily explained by the observation that, contrary to the non-batched case, now a parameter loaded in cache is re-used for each of the vectors in the batch, before being trashed. Unfortunately, batching rises significantly the mean response time of a NN, since the result of a NN inference can be provided only after the entire batch has finished processing.

\section{NEURAL NETWORK SPLIT}

The runtime analysis of AlexNet shows that CPUs are efficient executors for conv, pool and norm layers, and far less efficient ones when it comes to the processing of fc layers. That is, the CPU's pipeline is stalled for a large fraction of time during the processing of fc layers, which effectively wastes otherwise useful computation power. Batching improves the situation, but it is a non viable approach for latency sensitive online workloads. 


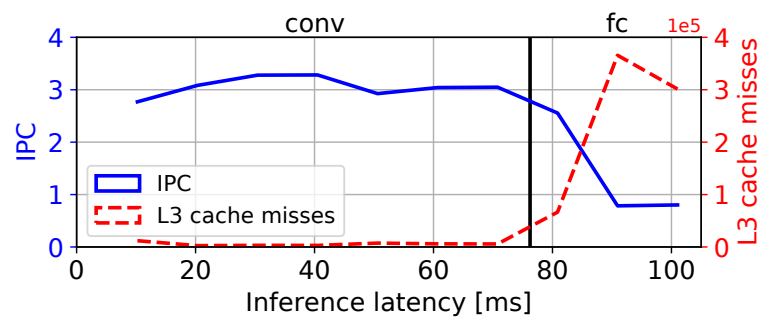

Figure 4: IPC and L3 cache misses

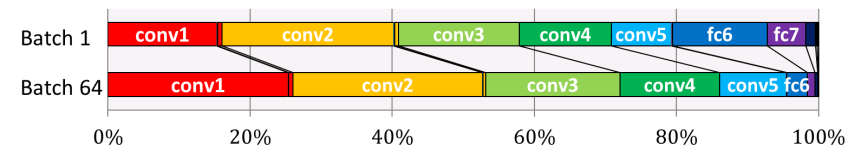

Figure 5: Normalized per-layer inference latency

Taking into account that NNs composed only of fc layers constitute the vast majority of NN workloads (cf. Sec.2), it is clear that developing a solution to improve the execution of these layers can provide important benefits. That is, a suitable executor for fc layers could on the one side lower the inference processing latency, and on the other side free CPUs resources that could be better used for other workloads ${ }^{1}$.

Before trying to understand whether programmable network devices could be such an efficient $f c$ layers executor, it is worth to evaluate and quantify the additional overheads that may be introduced by a split of the NN processing between two different executors. This will help in identifying the issues in using current accelerators.

In particular, we first verify if splitting the execution among two executors has an impact on the processing efficiency, e.g., due to potential cold cache effects or for the inability to leverage some data pre-fetching. Then, we quantify the cost introduced by additional data movements between executors.

\subsection{Split processing overhead}

To evaluate the impact on the processing efficiency, we select two homogeneous executors, but with independent memories and caches, i.e., the two CPUs of our NUMA machine. Being able to exchange data using the fast QPI interconnection between their sockets, using the CPUs minimizes the overhead of data movements. Moreover, since we use homogeneous executors, we can directly compare the measurements for a split execution to the measurements of a non-split one.

To instrument our testbed, we modify our NN implementation to spawn two processes, each one executing a portion of the NN. The first process performs its layers processing up to the split point, then transfers the intermediate result to the second process that continues the processing till the end of the NN. Both processes load

${ }^{1}$ In effect, stalls may be mitigated by performing a context switch while waiting for the data to be loaded, however this would require sharing the CPU with other tasks. This is likely to increase the processing latency, and could be therefore not viable for latency sensitive workloads. Regardless, since the L3 cache is anyway shared, sharing the CPU with other tasks may in fact be counterproductive.
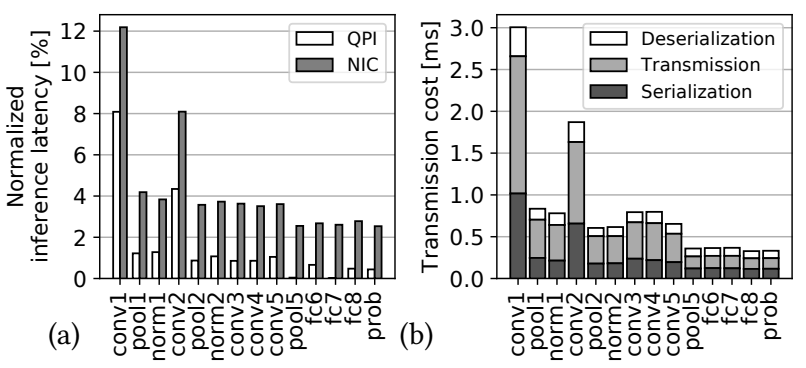

Figure 6: Normalized inference latency and communication overhead breakdown for different split points

the network model in their respective NUMA node's local RAMs. QPI bars of Fig. 6a show that there is an overhead in splitting the NN execution, which can cause up to $8 \%$ higher inference time when compared to the non split execution case, and almost no increases in some cases. The overhead is higher when the splitting happens after a layer with a large output vector.

The test results suggest that the overhead is dominated by the need to wait for the loading of the input vector in the cache of the second CPU. This explanation is easily verified noticing that the overhead of splitting the execution before layers with smaller inputs is always within a modest $1.3 \%$.

\subsection{Communication overhead}

In this second test, we split again the execution of the processing between the two CPUs of our NUMA machine, however, this time we force the communication between them to happen through the NIC. That is, we connect the NIC's ports together with a cable in a loop, and modify our NN implementation to transfer the intermediate data (at the split point) using a TCP connection. To avoid the overheads of connection establishment and TCP slow-start, the connection is pre-opened and configured with a very large initial congestion window.

As expected, the end-to-end processing latency grows. NIC bars of Fig. 6a show that the latency increase ranges between $2-12 \%$ and follows a pattern similar to the one of QPI bars.

Fig. $6 \mathrm{~b}$ shows the breakdown of the overhead due to communication in three components: (i) data serialization time, required to prepare the intermediate result for transmission; (ii) transfer time, needed to send the data over the TCP socket and to receive it at the other end, e.g., it includes the time to transfer data through the PCIe bus and the NIC's ports; (iii) data deserialization time, required to prepare the received data for feeding it to the second portion of the NN. Of the three components, the transmission time is significantly big only when the split happens after a layer with a large output vector, otherwise it only contributes from tens to hundreds of microseconds. The combination of serialization and deserializaton times, instead, always provide about 300us of additional latency, which may be significant on smaller networks. For instance, the three $\mathrm{fc}$ layers at the end of the network take about $19 \mathrm{~ms}$ in total for processing, therefore an additional ms would correspond to about a $1.5 \%$ latency increase. 


\subsection{Takeaway}

The tests presented in this section confirm and clarify the issues in using current accelerators for performing $\mathrm{NN}$ inference. On the one side, the overhead of splitting a NN execution can be limited if the split point is carefully selected. This suggests that using an accelerator to perform part of the NN processing is possible. On the other side, the cost of moving data to the accelerator may be significant, in particular for smaller networks, confirming the numbers reported in [9].

\section{BaNaNa SPLIT}

The results presented in previous sections highlight that the overhead of moving data is a big issue when using off-path accelerators, such as GPGPUs or TPUs. However, if the accelerator is implemented within the network devices that are already on path, such extra data movement is not required.

That is, a data transmission from the CPU/memory to the NIC (and then to the switches) is anyway required, in order to send the $\mathrm{NN}$ processing result back to the requester (e.g., a service front-end). Thus, if on-path network devices could perform NN processing, they would introduce mainly the overhead due to the splitting of the processing. Such overhead, as we have seen, can be very little if the split is carefully done, i.e., taking into account layer input sizes. But can SmartNICs and programmable switches do NNs processing?

The hardware architecture of these devices certainly include large amounts of fast SRAM that could hold the NN's parameters (or part of them). However, they are usually designed to perform only simple operations, which do not include the relatively complex arithmetic operations required by a NN.

To overcome this issue, we introduce BaNaNa Split. Our approach leverages recent advances in NNs research, which show that in many cases the fc layers of a NN can be simplified through a process called quantization, without sacrificing much of the model accuracy $[7,11,18]$. A quantized model reduces the number of bits used to represent a NN's activations and parameters and is therefore smaller in size than its original version. This helps in fitting the model parameters in a suitably large SRAM. Moreover, some quantization techniques enable the use of simpler arithmetic operations, which can be supported by current network devices [17].

Therefore, given a NN model, BaNaNa Split works as follows. At configuration time, it splits the model right before the fc layers. The first set of layers is going to be executed e.g., on a CPU. The remaining layers undergo a quantization process that actually transforms the original NN model into a form that can be executed on network devices properly programmed for the task. At runtime, a NN inference request is first handled by a machine's CPU, then, the intermediate result is encapsulated in network packets (e.g., in a header) and sent to the machine's (Smart)NIC. Here, the intermediate result is processed by the NIC to execute the quantized layers. Finally, the quantized layers output is encapsulated again in the network packet, and then sent to its final destination.

Since the intermediate results are encapsulated in the packets, BaNaNa Split can potentially distribute the computation to different devices on-path, including both SmartNICs and programmable switches.

\subsection{Implementation}

Our proof-of-concept prototype is designed to work with existing commercial network devices. It uses binarization as quantization technique, i.e., both activations and parameters are represented using 1 b. The resulting $\mathrm{NN}$ is called a Binary Neural Network $(\mathrm{BNN})^{2}$ and only requires bitwise logic operations and population count computation to be implemented $[7,11,18]$. Thus, the implementation of a BNN processing can be supported by current SmartNICs and programmable switches already. In particular, we use N2Net [21] to implement BNNs on a programmable switch, and further extend it to add network processor-based SmartNICs support. $\mathrm{N} 2 \mathrm{Net}$ is a compiler that given a BNN model description generates a $\mathrm{P} 4$ [1] program for the configuration of an RMT-like switch pipeline [2]. Our extension for SmartNICs replaces the N2Net's popcount implementation to leverage the built-in support for such operation, when available ${ }^{3}$.

Early tests. At the time of writing, we are still in the process of building a fully-fledged end-to-end prototype. Thus, here we provide just the results of a micro-benchmark that evaluates the execution of a single (binarized) fc layer with 4096 neurons, using a network processor-based SmartNIC.

The activation vector for such layer contains $4096 \mathrm{~b}$, i.e., 512B, and is therefore encoded in a single network packet. The layer's parameter size is $2 \mathrm{MB}$, and the parameters are pre-configured before running the execution. Here, recall that high-performance network devices do not typically use cache hierarchies for memory serving packet processing, in order to provide fast and guaranteed memory access times. Under these conditions, our SmartNIC implementation takes 1 millisecond to process the layer.

For the switching chip evaluation, we only performed an estimation based on the RMT architecture, and based on the numbers reported in [21]. In such case, we assume the switch can execute at most 96 neurons in parallel, during the processing of a single packet. In effect, this would require the recirculation of the packet 43 times to execute the 4096 neurons of the layer. Unfortunately, we cannot directly link these numbers to the processing latency, since we could not test yet our prototype on a hardware programmable switch.

\section{DISCUSSION}

Several works are exploring the suitability of programmable network devices to assist the processing of different types of workloads [3, 8, 13-15, 19, 20]. BaNaNa Split adds to this list, exploring the suitability of current programmable network devices to work as $\mathrm{NN}$ accelerators.

Our work, however, is necessarily built on important assumptions. In first place, we heavily rely on quantized NN models. While promising, these models are still heavily research-oriented and their widespread applicability is not demonstrated yet. Thus, the success of these approaches is likely to have a major impact on the possibility to use (current) networking hardware as NN accelerators. Second, we currently assume we can fit an entire layer's parameters in a network device's fast memory. Even if this is the case with a quantized model, we are not taking into account the traditional

\footnotetext{
${ }^{2}$ Hence the name BaNaNa Split.

${ }^{3}$ The POPCNT is actually described by means of a P4's extern action.
} 


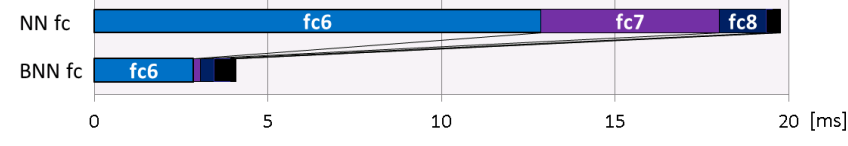

Figure 7: BNN fc execution latency

networking functions that make use of the very same memory. To mitigate this issue it is possible to distribute the $\mathrm{NN}$ computation between NICs and multiple on-path switches.

Another important observation is that there is a subtle side-effect in using quantized models. That is, being smaller they can likely improve the execution efficiency on CPUs, reducing the need for acceleration. Fig. 7 shows this effect by presenting the processing time for the AlexNet's fc layers when their size is reduced to the size of binarized layers. The processing time is reduced to the $21 \%$ of the original processing time, thus reducing the potential benefits that would be introduced by a solution like $\mathrm{BaNaNa}$ Split. Nonetheless, considering the size of the workloads (cf. Sec 2), even after the reduction the share of workload that could be offloaded from the CPUs remains significant.

In terms of related work, it should be noted that Microsoft's BrainWave [16] uses network-attached FPGAs to accelerate NNs. $\mathrm{BaNaNa}$ Split approach is different in that it uses the very same network device hardware that does packet processing to also perform NN processing, while in our understanding BrainWave could be considered a dedicated accelerator deployed "close to" the network.

\section{CONCLUSION}

While far from a complete system implementation, our study and early prototype show that some workloads could indeed be potentially offloaded to network devices, and that there are reasons to do so, especially when end-to-end processing latency is a concern. In particular, CPUs speed is limited by memory access times when processing a NN's fully connected layers. Thus, a large fraction of the CPU compute power is wasted with stalled cycles. Offloading the execution of such layers to network devices could improve the efficiency of the overall infrastructure, freeing CPUs for more compute-intensive workloads. Nonetheless, the applicability of the BaNaNa Split approach still requires addressing open challenges both in the research of optimized neural network models and in network device architectures.

\section{ACKNOWLEDGMENTS}

This work has received funding from the European Union's Horizon 2020 research and innovation programme under grant agreement No. 761493 ("5GTANGO"). This paper reflects only the authors' views and the European Commission is not responsible for any use that may be made of the information it contains.

\section{REFERENCES}

[1] Pat Bosshart, Dan Daly, Glen Gibb, Martin Izzard, Nick McKeown, Jennifer Rexford, Cole Schlesinger, Dan Talayco, Amin Vahdat, George Varghese, et al. 2014. P4: Programming protocol-independent packet processors. ACM SIGCOMM CCR 44, 3 (2014), 87-95.

[2] Pat Bosshart, Glen Gibb, Hun-Seok Kim, George Varghese, Nick McKeown, Martin Izzard, Fernando Mujica, and Mark Horowitz. 2013. Forwarding Metamorphosis: Fast Programmable Match-action Processing in Hardware for SDN. In Proceedings of the ACM SIGCOMM 2013 Conference on SIGCOMM (SIGCOMM '13). ACM, New York, NY, USA, 99-110. https://doi.org/10.1145/2486001.2486011

[3] Huynh Tu Dang, Marco Canini, Fernando Pedone, and Robert Soulé. 2016. Paxos Made Switch-y. SIGCOMM Comput. Commun. Rev. 46, 2 (May 2016), 18-24. https://doi.org/10.1145/2935634.2935638

[4] Nikos Hardavellas. 2012. The rise and fall of dark silicon. USENIX ;login: 37 (2012), 7-17.

[5] Johann Hauswald, Yiping Kang, Michael A Laurenzano, Quan Chen, Cheng Li, Trevor Mudge, Ronald G Dreslinski, Jason Mars, and Lingjia Tang. 2015. DjiNN and Tonic: DNN as a service and its implications for future warehouse scale computers. In ACM SIGARCH Computer Architecture News, Vol. 43. ACM, 27-40.

[6] K. Hazelwood, S. Bird, D. Brooks, S. Chintala, U. Diril, D. Dzhulgakov, M. Fawzy, B. Jia, Y. Jia, A. Kalro, J. Law, K. Lee, J. Lu, P. Noordhuis, M. Smelyanskiy, L. Xiong, and X. Wang. 2018. Applied Machine Learning at Facebook: A Datacenter Infrastructure Perspective. In 2018 IEEE International Symposium on High Performance Computer Architecture (HPCA). 620-629. https://doi.org/10.1109/HPCA.2018.00059

[7] Itay Hubara, Matthieu Courbariaux, Daniel Soudry, Ran El-Yaniv, and Yoshua Bengio. 2016. Binarized Neural Networks. In Proceedings of the 30th International Conference on Neural Information Processing Systems (NIPS'16). Curran Associates Inc., USA, 4114-4122.

[8] Xin Jin, Xiaozhou Li, Haoyu Zhang, Robert Soulé, Jeongkeun Lee, Nate Foster, Changhoon Kim, and Ion Stoica. 2017. NetCache: Balancing Key-Value Stores with Fast In-Network Caching. In Proceedings of the 26th Symposium on Operating Systems Principles (SOSP '17). ACM, New York, NY, USA, 121-136. https://doi. org $/ 10.1145 / 3132747.3132764$

[9] Norman P Jouppi, Cliff Young, Nishant Patil, David Patterson, Gaurav Agrawal, Raminder Bajwa, Sarah Bates, Suresh Bhatia, Nan Boden, Al Borchers, et al. 2017. In-datacenter performance analysis of a tensor processing unit. In Proceedings of the 44th Annual International Symposium on Computer Architecture. ACM, 1-12.

[10] Vadim Karpusenko, Andres Rodriguez, Jacek Czaja, and Mariusz Moczala. 2016. Caffe $e^{*}$ Optimized for Intel Architecture: Applying Modern Code Techniques. Technical Report. Intel.

[11] Minje Kim and Paris Smaragdis. 2016. Bitwise Neural Networks. CoRR abs/1601.06071 (2016). arXiv:1601.06071 http://arxiv.org/abs/1601.06071

[12] Alex Krizhevsky, Ilya Sutskever, and Geoffrey E Hinton. 2012. Imagenet classification with deep convolutional neural networks. In Advances in neural information processing systems. 1097-1105.

[13] Xiaozhou Li, Raghav Sethi, Michael Kaminsky, David G. Andersen, and Michael J. Freedman. 2016. Be Fast, Cheap and in Control with SwitchKV. In 13th USENIX Symposium on Networked Systems Design and Implementation (NSDI 16). USENIX Association, Santa Clara, CA, 31-44. https://www.usenix.org/conference/nsdi16/ technical-sessions/presentation/li-xiaozhou

[14] Ming Liu, Liang Luo, Jacob Nelson, Luis Ceze, Arvind Krishnamurthy, and Kishore Atreya. 2017. IncBricks: Toward In-Network Computation with an In-Network Cache. In Proceedings of the Twenty-Second International Conference on Architectural Support for Programming Languages and Operating Systems. ACM, 795-809.

[15] Rui Miao, Hongyi Zeng, Changhoon Kim, Jeongkeun Lee, and Minlan Yu. 2017. SilkRoad: Making Stateful Layer-4 Load Balancing Fast and Cheap Using Switching ASICs. In Proceedings of the Conference of the ACM Special Interest Group on Data Communication (SIGCOMM '17). ACM, New York, NY, USA, 15-28. https://doi.org/10.1145/3098822.3098824

[16] Microsoft. 2017. Microsoft unveils Project Brainwave for realtime AI. https://www.microsoft.com/en-us/research/blog/ microsoft-unveils-project-brainwave/

[17] Daisuke Miyashita, Edward H Lee, and Boris Murmann. 2016. Convolutional neural networks using logarithmic data representation. arXiv preprint arXiv:1603.01025 (2016)

[18] Mohammad Rastegari, Vicente Ordonez, Joseph Redmon, and Ali Farhadi. 2016. XNOR-Net: ImageNet Classification Using Binary Convolutional Neural Networks. CoRR abs/1603.05279 (2016). arXiv:1603.05279 http://arxiv.org/abs/1603. 05279

[19] Amedeo Sapio, Ibrahim Abdelaziz, Abdulla Aldilaijan, Marco Canini, and Panos Kalnis. 2017. In-Network Computation is a Dumb Idea Whose Time Has Come. In Proceedings of the 16th ACM Workshop on Hot Topics in Networks, Palo Alto, CA, USA, HotNets 2017, November 30 - December 01, 2017. 150-156. https://doi. org/10.1145/3152434.3152461

[20] Naveen Kr. Sharma, Antoine Kaufmann, Thomas Anderson, Arvind Krishnamurthy, Jacob Nelson, and Simon Peter. 2017. Evaluating the Power of Flexible Packet Processing for Network Resource Allocation. In 14th USENIX Symposium on Networked Systems Design and Implementation (NSDI 17). USENIX Association, Boston, MA, 67-82. https://www.usenix.org/conference/nsdi17/ technical-sessions/presentation/sharma

[21] Giuseppe Siracusano and Roberto Bifulco. 2018. In-network Neural Networks. arXiv preprint arXiv:1801.05731 (2018). 